\section{Prostata: Erkrankte Brüder steigern Risiko}

Das Auftreten eines Prostatakarzinoms bei Verwandten ersten Grades ist immer ein Alarmsignal. Neue Ergebnisse, die das eindringlich unterstreichen, liefert eine große schwedische Datensammlung zur familiären Krebsbelastung.

E ine familiäre Häufung von Prostata-

E karzinomen ist zwar schon lange bekannt, doch ließen sich bisher keine Hochrisiko-Suszeptibilitätsgene identifizieren. Dem Erkrankungsrisiko in Abhängigkeit von Zahl und Alter der Verwandten ersten Grades mit Prostatakarzinom ging eine schwedische Analyse nach. Sie nutzte Daten des schwedischen Krebsregisters, des sog. Multigenerationenregisters mit Angaben über die familiären Beziehungen sowie des Registers der Todesursachen. Mit 26.651 Prostatakrebs-Patienten insgesamt, davon 5.623 mit familiärer Belastung, war es die bisher größte Studie zu dieser Frage.

Das Risiko, an Prostatakrebs zu erkranken, stieg mit der Zahl betroffener Verwandter ersten Grades und sank mit zunehmendem Alter. Am höchsten war es für Männer unter 65 Jahren mit drei betroffenen Brüdern (Hazard Ratio etwa 23), am geringsten für Männer zwischen 65 und 74 Jahren mit einem betroffenen
Vater (HR etwa 1,8). Ein Bruder mit Prostatakarzinom bedeutete ein höheres Erkrankungsrisiko als ein erkrankter Vater; jeder erkrankte Bruder mehr sorgte für einen weiteren Anstieg des Risikos. Auch das Alter bei Diagnose spielte eine Rolle: Je jünger, desto höher das Risiko für die Verwandten ersten Grades. Ein ähnliches Muster wie bei der Inzidenz fand sich bei der Mortalität.
Fazit: Die exakte Erfassung des familiären Risikos ist wichtig, um eine vernünftige Basis für klinische Beratung und Screeningempfehlungen zu haben. Die schwedischen Daten zeigen, dass das Erkrankungsrisiko bei mehreren Verwandten ersten Grades mit Prostatakarzinom enorm zunimmt. In diesem Fall sollte möglicherweise ebenso wie bei Erkrankung eines Verwandten im Alter unter 65 Jahren früher als sonst üblich ein Screening ins Auge gefasst werden. uwe

Brandt A et al. Age-specific risk of incident prostate cancer and risk of death from prostate cancer defined by the number of affected family members. Eur Urol 2010; 58: 275-80.

\begin{tabular}{l|r|r|r|r|}
\multicolumn{4}{|c|}{ Mortalitätsrisiko erhöht sich mit der Anzahl erkrankter Verwandter } & Tabelle \\
\hline An Pankreaskrebs erkrankte Verwandte & Anzahl & Hazard Ratio & 95\%-Konfidenzintervall \\
\hline Keine & 2.113 & - & - \\
\hline Vater & 306 & 1,81 & $1,61-2,04$ \\
\hline Ein Bruder & 139 & 2,75 & $2,32-3,26$ \\
\hline Vater und ein Bruder & 24 & 2,96 & $1,98-4,43$ \\
\hline Zwei Brüder & 15 & 6,29 & $3,79-10,46$ \\
\hline Vater und zwei Brüder & 5 & 9,74 & $4,05-23,43$ \\
\hline Drei Brüder & 2 & 8,12 & $2,03-32,50$
\end{tabular}

